\title{
Desquamative Inflammatory Vaginitis: The Unknown
}

\author{
María Trinidad Alumbreros Andújar ${ }^{*}$, Ana González López ${ }^{1}$, Celia Pérez Parra ${ }^{1}$, Rafael López Pérez ${ }^{2}$, \\ Carmen Céspedes Casas ${ }^{1}$, María Mercedes Ramírez Gómez ${ }^{1}$, Castor Martin Francisco ${ }^{1}$, Francisco \\ Javier Haya Palazuelos ${ }^{1}$
}

\begin{abstract}
Introduction: Desquamative inflammatory vaginitis (DIV) is a chronic inflammatory process of unknown etiology, characterized by genital pain and profuse vaginal discharge, mainly affecting perimenopausal women. It is an entity little known by clinicians, leading to a delay in diagnosis and the consequent alteration of the patients' quality of life. The aim in this manuscript is to analyze DIV through the review of a case and the scientific literature.

Case presentation: We report the case of a 40 -year-old woman who presented this clinical profile for several years, being misdiagnosed as more common syndromes such as infectious vaginitis and vaginismus. Finally, after the diagnosis of DIV and the establishment of the specific treatment, she showed a clear symptomatic improvement.

Conclusion: The prevalence of DIV is unknown and underestimated, and may affect $8 \%$ of women with vulvovaginal symptoms. To reach the correct diagnosis, it is required to observe a few simple clinical and laboratory criteria defined by Sobel in 1994. It responds to the topical therapy with clindamycin or hydrocortisone in $95 \%$ of cases, but in most cases a long-term maintenance therapy is necessary to control the symptoms.

Keywords: Dyspareunia, Inflammation, Vaginitis
\end{abstract}

\section{Introduction}

The vaginal symptoms such as profuse vaginal discharge or dyspareunia are the most common reasons to visit the gynaecologist. However, in most cases, they are treated empirically, leading to diagnosis of the most frequent causes of vaginitis such as infectious ones (1).

The symptoms perpetuate in time with no improvement at a low percentage of women, affecting their quality of life. It is then that the clinician has to maintain a high clinical suspicion and use the necessary tests to attain the correct diagnosis.

This is the case of a patient who suffered severe incapacitating dyspareunia for a long time. Finally, she was correctly diagnosed as desquamative inflammatory vaginitis (DIV) and the specific treatment for her was prescribed. Consequently, there was a clear symptomatic improvement.

\section{Case presentation}

A 40-year-old woman presented to our unit complaining mostly about three years of dyspareunia, as well as postcoital dysuria, genital dryness and pruritus of variable acuteness.

She had no medical-surgical history of interest. She was nonsmoker and did not have any other toxic habits. Among her gyneco-obstetric records, it was stated that the patient was normorrheic, had a delivery by caesarean section and a stable partner for 22 years and presented as secondary sterility. She had come to the clinic for the same reason several times, showing no improvement to the different prescribed treatments: anti-inflammatory drugs, cicatrizant and hydrating creams, lubricants, topical estrogens, topical antifungal medication and probiotics.

Physical and colposcopic examination revealed a normal vulva and a vagina with a moderate quantity of yellowish discharge of unspecific characteristics. In the vaginal sidewalls several over-raised erythematous patches of small size were observed in the lower third part and introitus. The cervix and the rest of the genital tract did not reveal any other pathological findings (Figure 1).

We performed the following complementary tests:

- A vulvovaginal culture, resulting negative.

- The vaginal $\mathrm{pH}$ was over 4.5.

- A cervical cytology that did not show any evidence of dysplasia. The polymerase chain reaction (PCR) for the Human Papilloma virus was negative.

- A vaginal biopsy which was reported as a mucous with superficial ulceration and acute unspecific chronic inflammation (follicular) with no significant dysplasia or malignancy. There were no findings with PAS staining (Figure 2). 


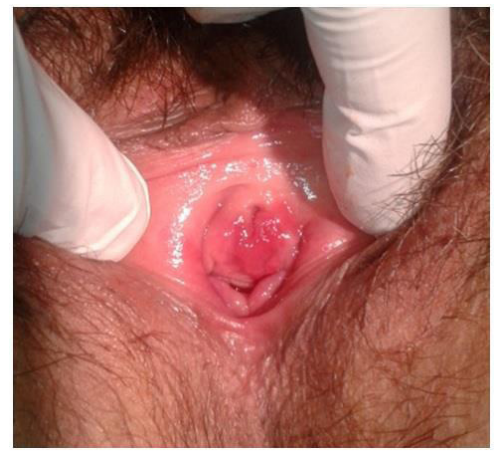

Figure 1. Erythematous Patch in the Vaginal Mucous Membrane and Vestibule.

We dismissed the infectious and neoplastic causes, as well as other common disorders such as lichen planus or pemphigus vulgaris and we thought that it could be DIV. We prescribed $2 \%$ clindamycin cream, 4 to 5 grams intravaginally, once daily for 4 weeks following with hydrocortisone ovules, 0.01 grams per ovule, once daily for 2 weeks. The patient showed a considerable clinical improvement in the follow-up examination after 4 months.

\section{Discussion}

The term DIV was first established by Gray and Barnes in 1965. However, it was not until 1994 that Sobel described the pathology in detail, as well as its treatment on 51 women, thereby determining the diagnostic criteria that are valid until the present (1).

It is a little-known entity and, therefore, its prevalence may be underestimated. It has been determined that it represents about $4.3 \%$ to $8 \%$ of all the vulvovaginal pathologies $(2,3)$.

It can present at any age, although it is much more frequent at perimenopause, being 48 the mean age in the largest case series published (2). In a small case-control study it was noticed that the patients who presented this pathology were older, with higher levels of education and had less high-risk sexual behaviours than the common population. Moreover, it was also observed that it occurred more frequently in women with previous vaginal infections and those with fertility problems and a low pregnancy rate (4). DIV is a chronic syndrome of unknown etiology. No pathogenic microbial cause has been identified; therefore, its pathophysiology seems to be more related to an inflammatory process rather than an infectious one. Some cases related to a deficiency of vitamin D (5) and the toxic shock syndrome produced by the Staphylococcus aureus have been described (6).

This pathology is characterized by the presence of genital pain, especially as dyspareunia or burning and itching, which can be incapacitating. It can additionally occur with a profuse yellowish discharge. An erythematous and ecchymotic vaginal mucous, which is painful at palpation, is revealed. The vestibule is also often affected (2). Those symptoms are often present long time before the correct diagnosis, since it is a little-known entity and the clini-

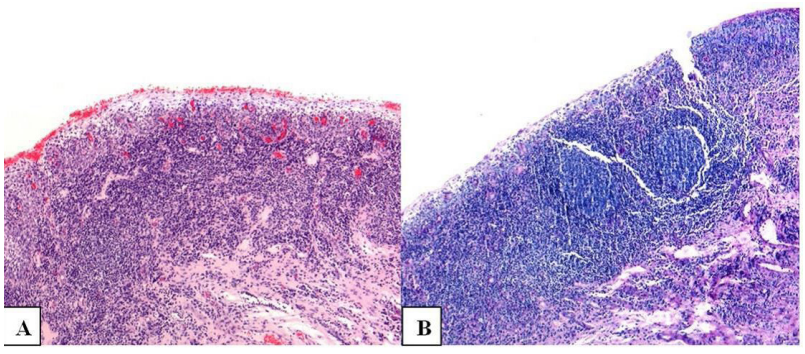

Figure 2. Biopsy From the Affected Vaginal Mucous. In A (Hematoxylin-eosin staining) we can observe a superficial ulceration and an inflammatory infiltrate with lymphocytic predominance. PAS staining (B) shows the presence of lymphoid follicles and the absence of yeast.

cian considers those symptoms as part of the clinical profile of other forms of vaginitis more common than DIV (candidiasis, bacterial vaginosis). In the largest case series published, patients suffered the symptoms above for an average of 15.5 months before being diagnosed as DIV (2). Not so long ago it was considered that several dermatological pathologies affecting the vulva and the vagina, such as lichen planus or pemphigus vulgaris could occur as DIV (7). However, nowadays DIV is considered as an independent entity of unknown cause with specific clinical diagnostic criteria. Those criteria were defined by Sobel in 1994. They all should be accomplished to get to diagnosis (1):

- At least one of the following symptoms: profuse vaginal discharge, dyspareunia, pruritus, burning or irritation.

- Vaginal mucous inflammation as erythema, ecchymosis and linear or focal erosions.

- Vaginal $\mathrm{pH}>4.5$.

- Saline microscopy revealing an increase of parabasal and inflammatory cells (leucocytes) with an inflammatory/epithelial cell ratio greater than $1 / 1$.

- Moreover, any vaginal infection with the specific cultures (trichomoniasis, yeast, gonococcus, chlamydia, and bacterial vaginosis) and all the immune dermatological pathologies with vaginal biopsy and immunofluorescence techniques should be excluded. The histological study on DIV usually shows a pattern of unspecific inflammation with a mixed infiltrate of lymphocytes, eosinophils and plasma cells (7).

- The main pathologies that have to be taken into account for the elaboration of a differential diagnosis are the following (3):

- Erosive lichen planus: Chronic dermatological disease of probable immune origin that affects skin and mucous, the vaginal mucous membrane, among others. It produces a grave inflammation on the whole vaginal mucous membrane, causing a very friable and bleeding epithelium in which synechiae may appear. For diagnosis it is important to examine the rest of mucous, especially the mouth and execute a biopsy containing healthy and affected mucous.

- Pemphigus vulgaris and cicatricial pemphigoid: They 
are immune pathologies that affect the mucous with blisters which turn into erosions. The pemphigoid can be crippling and cause severe scarring. For diagnosis it is vital to explore the other mucous and perform the biopsy with immunofluorescence techniques.

- Severe atrophic vaginitis: This pathology is common in menopausal women with hypoestrogenism. A decrease of epithelial cells and lactobacilli is present, as in DIV. Nevertheless, in this case there is a clear improvement of the symptomatology to the local estrogens administration.

- Vestibulodynia: If the clinician commits the mistake of not examining the vagina in a woman with dyspareunia and vestibular pain may disregard DIV and misdiagnose the patient as vestibulodynia.

Regarding the treatment for DIV, there are no randomized clinical trials of different therapeutic options. However, using 2\% clindamycin intravaginally, daily for 4-6 weeks there is a symptomatic improvement mostly in more than $95 \%$ of cases $(1,2)$. Another therapeutic option is the daily administration of $10 \%$ hydrocortisone intravaginally. There is no study which compares this treatment with clindamycin. Nonetheless, the good response of both of them makes us think about the non-infectious etiology of the syndrome, since clindamycin has a recognized anti-inflammatory effect which works by means of the pro-inflammatory cytokine synthesis inhibition (IL-1, 6, 8 and TNF- $\alpha$ ) (2). In a small study $150 \mathrm{mg}$ of oral clindamycin were used twice per week along with intravaginal clobetasol propionate and weekly fluconazole as prophylaxis of candidiasis. It was noticed that the improvement rate was lower than using topical clindamycin. As a consequence, all this supports the local anti-inflammatory effect of the latter (7).

Either of the 2 treatment procedures led to a dramatic and fast symptomatic improvement; therefore, the absence of improvement would make vital to reconsider diagnosis. However, recurrence is frequent, up to a rate of $30 \%$ (2). For that reason, long-term maintenance treatment is necessary in most patients. It is advisable that the patient uses the suitable dose to maintain her asymptomatic. The topical estrogen administration may be useful to avoid relapse, since the lactobacilli colonization is increased and the vaginal epithelium is strengthened (3).

\section{Conclusion}

DIV is an entity little known by clinicians. Nevertheless, it can represent $8 \%$ of the vulvovaginal pathology in the common population, causing incapacitating symptoms for a long time. A high clinical suspicion is necessary to elaborate both an early diagnosis and treatment and improve the patients' quality of life.

\section{Ethical issues}

The patient has not been identified with the provided information, including both written descriptions and photographic depictions. Authors are responsible for ensuring compliance with relevant privacy regulations and for obtaining release for confidential information. The written informed consent was obtained from the patient.

\section{Financial Support}

There has been no financial support for the realization of this manuscript.

\section{Conflict of Interests}

No potential conflict of interest relevant to this article was reported.

\section{Acknowledgments}

We would like to thank the Department of Pathology for their collaboration in the elaboration of this manuscript.

\section{References}

1. Sobel JD. Desquamative inflammatory vaginitis: a new subgroup of purulent vaginitis responsive to topical 2\% clindamycin therapy. Am J Obstet Gynecol. 1994;171(5):1215-1220.

2. Sobel JD, Reichman O, Misra D, et al. Prognosis and treatment of desquamative inflammatory vaginitis. Obstet Gynecol 2011;117(4):850-855. doi:10.1097/ AOG.0b013e3182117c9e.

3. Stockdale CK. Clinical spectrum of desquamative inflammatory vaginitis. Curr Infect Dis Rep. 2010;12(6):479-83. doi:10.1007/s11908-010-0135-y.

4. Newbern EC, Foxman B, Leaman D, Sobel JD. Desquamative inflammatory vaginitis: an exploratory case-control study. Ann Epidemiol. 2002;12(5):346352.

5. Peacocke M, Djurkinak E, Thys-Jacobs S. Treatment of desquamative inflammatory vaginitis with vitamin D: A case report. Cutis. 2008;81(1):75-78.

6. Pereira N, Edlind T, Schlievert PM, Nyirjesy P. Vaginal toxic shock react in triggering desquamative inflammatory vaginitis. J Low Genit Tract Dis. 2013;17(1):88-91. doi:10.1097/ LGT.0b013e3182656991.

7. Murphy R, Edwards L. Desquamative inflammatory vaginitis: what is it? J Reprod Med. 2008;53(2):124128.

Copyright $\odot 2015$ The Author(s); This is an open-access article distributed under the terms of the Creative Commons Attribution License (http://creativecommons.org/licenses/by/4.0), which permits unrestricted use, distribution, and reproduction in any medium, provided the original work is properly cited. 